\title{
Knowledge, Attitude and Perceived Barriers towards practice of Evidence Based Dentistry among Indian postgraduate dental students
}

\author{
Prabhu. $\mathrm{S}^{1}$, Joseph John ${ }^{2}$, Saravanan. $\mathrm{S}^{3}$ \\ ${ }^{1}$ Post - Graduate, Department of Public Health Dentistry, Saveetha Dental College \& Hospital, India \\ ${ }^{2}$ Professor and Head, Department of Public Health Dentistry, Saveetha Dental College \& Hospital, India \\ ${ }^{3}$ Professor, Department of Public Health Dentistry, Saveetha Dental College \& Hospital, India
}

\begin{abstract}
Objective- To assess the knowledge, attitude and perceived barriers towards practice of evidence based dentistry among Indian postgraduate dental students.

Method-This is a cross-sectional questionnaire study. 234 post-graduate dental students participated in the present study (response rate - 86.7\%). Knowledge, attitude and perceived barriers towards practice of evidence based dentistry was assessed using a validated, self-administered questionnaire. Frequency tables were computed. Independent samples $t$ - test was used to test for the difference in mean knowledge scores between clinical and non-clinical department $(p<0.05)$ is considered statistically significant.

Results- 65 (27.8\%) subjects were not familiar with evidence based dentistry, Mean knowledge reagarding terminologies used in evidence based dentistry was high among clinical than non-clinical students. A positive attitude was seen among the post graduates in practice of evidence based dentistry, there exist certain barriers in practice of evidence based dentistry.

Conclusion- The postgraduates although familiar with the sources of evidence based dentistry, their knowledge of the terms used in evidence based dentistry is limited a positive attitude is seen among respondents towards imparting evidence based dentistry in clinical practice and Potential barriers still exist in practice of evidence based dentistry.
\end{abstract}

Keywords: Dentistry, Dental education, Evidence-Based Dentistry, Postgraduate students

\section{Introduction}

It is generally accepted that the more experience a physician or a dentist possess, better the quality of health care delivery. However recent studies had shown that there is in fact an inverse relationship between the number of years of practice and the quality of care provided ${ }^{[1]}$, this is because a dentists 'toolkit' is created during training and is not updated regularly, practice innovations that involve theoretical shifts may be harder to incorporate into the practice of dentists who had been trained a long time ago.

The practice of dentistry is becoming more complex and challenging. Changing socio-economic patterns, knowledgeable health care consumers, rapid technical advances and the information 'explosion' all place greater demands on clinical decision making ${ }^{[2]}$. The American Dental Association defined Evidence Based Dentistry as an approach to oral healthcare that requires the judicious integration of systematic assessment of clinically relevant scientific evidence relating to the patients oral and medical conditions and history, with the dentists clinical expertise and the patients treatment needs and preferences ${ }^{[3]}$.

Evidence based dental practice is said to be the current best approach to provide interventions, the advantages of evidence based practice are that they were scientifically proven to be safe, efficient and costeffective (e.g. recommendations from systematic reviews) ${ }^{[4,5]}$, The reasons for this are assumed to be through improvements in physicians' and dentists' skills and knowledge, as well as in the communication between patients and their physicians about the rationale behind clinical recommendations made ${ }^{[4,6,7 \text { and } 8]}$.

Evidence based dentistry serves to reduce the variation in opinion between dentists in the diagnosis, prognosis, treatment outcomes and cost of care for patient with similar diseases, it depends on four different factors, 1. Quality of science underlying clinical evidence, 2. Quality in making clinical decisions, 3. Variations in the level of clinical skill and 4. Large and increasing volume of literature ${ }^{[9]}$.

Principles and methods of evidence-based dentistry give dentists an opportunity to apply relevant research findings to the care of their patients. Evidence based dentistry may ultimately protect dental practitioners from litigation as practitioners who take an evidence-based approach to care will be able to provide evidence trails for their clinical decision making ${ }^{[9]}$.

Dental education and dental care delivery systems are greatly improved in India due to increased dental health workforce and development in field of dental research, However dental graduation training program in India is mainly targeted towards preventive and curative dental procedures, there is a lack of emphasis on the 
application of evidence based dentistry in clinical practice. On the other hand, the term evidence based dentistry is widely used, but not widely understood among post-graduates due to lack of in depth training to distinguish good science from poor science. Most of the post-graduate dental students' clinical questions and problems are solved by a combination of instructors intuition, training and clinical experience, which may or may not be based on scientific evidence. This type of training which relies heavily on clinical experience and information learned in dental school, seminars or from colleagues can lead to inappropriate treatment outcomes ${ }^{[10]}$.

Self-search of articles of relevance, and getting research into learning and subsequently into practice. Thus encouraging creativity into our defunct teaching system will be our saviour; this creativity must come in form of Evidence Based Education ${ }^{[11]}$. It is high time to assess the knowledge, attitude of post graduates towards the concept of evidence based dentistry to diagnose, treat, and prevent disease by translation of best scientific evidence into clinical practice. Post-graduates need to be prepared on evidence based dentistry not only for the benefit of their patients but also to improve the quality of research works carried out by them, the present day post-graduate students are going to be the future academicians, the knowledge, attitude and practice of this future academicians towards evidence based dentistry will be reflected in the quality of dental care provided to the people. There is a lack of evidence from dental literature regarding knowledge, attitude and perceived barriers towards practice of evidence based dentistry among the post-graduate dental students. Hence the aim of this study is to assess the knowledge, attitude and perceived barriers towards practice of evidence based dentistry among Indian postgraduate dental students.

\section{Materials and Methods}

Sample size required for the present study was calculated to be $\mathrm{N}=220$, with $80 \%$ power at $5 \% \alpha$ error, based on the studies conducted by I.M. Haron et al (2012) ${ }^{[12]}$ and Risahmawati RM Risahmawati et al $(2011)^{[13]}$. This cross-sectional study was conducted among three dental colleges chosen at random from the list of dental colleges in the state of Tamil Nadu, India. Each dental college formed the cluster and the postgraduate dental students from all the nine specialties in dentistry were invited to participate in the present study. Post graduate students enrolled since 2010 and who are willing to participate in the present study were included and Post-graduates who had already participated in any previous studies of such kind were excluded. Prior to the start of the study ethical approval was obtained from Institutional Ethics Committee, Saveetha University. Approval from the concerned dental college authorities and written informed consent from the post-graduates were obtained prior to the study.

A total of 270 survey forms were distributed among the post graduate dental students. The survey form consisted of Knowledge, attitude and perceived barriers towards practice of evidence based dentistry was assessed by employing a pre-tested, validated, self-administered questionnaire containing demographic information followed by a closed ended questionnaire previously used to assess knowledge, attitude and perceived barriers towards practice of evidence based dentistry in the study by Hendricson WD et al (2011) ${ }^{[14]}$ and Risahmawati RM Risahmawati (2011) ${ }^{[13]}$ was used. Questionnaire contained items on Familiarity with Evidence Based Dentistry sources, level of understanding of the common terms used in the evidence based dentistry papers, Attitude towards practice of evidence based dentistry and possible barriers towards practice of evidence based dentistry. Filled questionnaires were collected using a drop box placed at a common place in the college (Library). Only completely filled forms were considered for analysis.

The data collected was analysed and tested for significance using statistical software packages, SPSS software for windows (version 16.0). Frequency tables were computed. Independent samples t- test was used to test for the difference in mean knowledge scores between clinical and non-clinical department $(p<0.05)$ is considered statistically significant.

\section{Results}

The response rate for the present study was 234 / 270 (86.7\%), all the collected forms were thoroughly screened for missing data and none of them were excluded. Table 1: Shows the characteristics of the study population, Among the 234 study subjects, 139 (59.4\%) were male and $95(40.6 \%)$ were female. 174 (74.36\%) belonged to clinical branches of dentistry and $60(25.64 \%)$ study subjects were from the non-clinical department. Clinical practice was present among 38 (16.2\%) study subjects and $196(83.8 \%)$ study subjects did not have clinical practice. 
Table 1: Characteristics of study population

\begin{tabular}{|l|l|c|c|}
\hline \multicolumn{1}{|c|}{ Characteristic } & \multicolumn{1}{|c|}{ Description } & N & \% \\
\hline \multirow{2}{*}{ Sex } & Male & 139 & 59.4 \\
\cline { 2 - 4 } & Female & 95 & 40.6 \\
\hline \multirow{2}{*}{ Age } & $\leq 26$ years & 138 & 58.97 \\
\cline { 2 - 4 } & $\geq 27$ years & 96 & 41.03 \\
\hline \multirow{2}{*}{ Department } & Clinical & 174 & 74.36 \\
\cline { 2 - 4 } & Non-clinical & 60 & 25.64 \\
\hline \multirow{2}{*}{ Clinical practice } & Yes & 38 & 16.2 \\
\cline { 2 - 4 } & No & 196 & 83.8 \\
\hline
\end{tabular}

Familiarity with the sources of evidence based dentistry among post-graduate dental students was shown in figure 1. $65(27.8 \%)$ subjects were unaware of evidence based dentistry, $72(30.8 \%)$ were unaware of cochrane database for systematic review and $24(10.3 \%)$ were unaware of pubmed/medline journal. Only 6 (2.6\%) subjects used Evidence based detistry in clinical practice to make decisions, 10 (4.2\%) subjects used cochrane database for systematic review in clinical decision making and $28(12 \%)$ subjects used Pubmed / Medline journals for clinical decision making.

\section{Figure 1 - Familiarity with the sources of Evidence Based Dentistry}

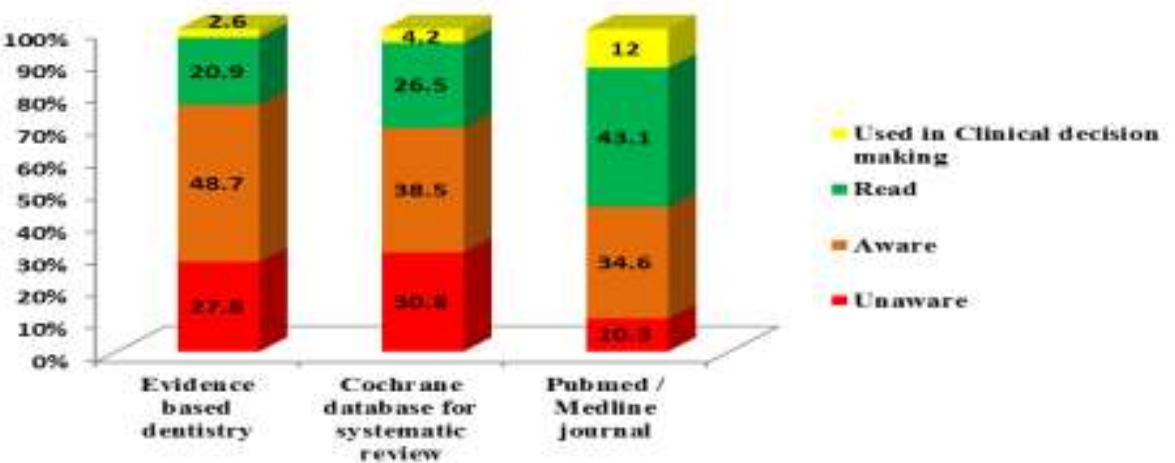

Knowledge regarding the terminologies used in evidence based dentistry was shown in table 2, mean knowledge regarding the terminologies were higher among clinical than non-clinical students, statistically significant difference was present between mean knowledge regarding the terminologies absolute risk and confidence interval $(\mathrm{p}<0.05)$. In certain terminologies the study subjects needed additional information as shown in table 3 .

Table 2 - Knowledge regarding the terminologies used in Evidence Based Dentistry

\begin{tabular}{|l|c|c|c|c|}
\hline \multirow{2}{*}{\multicolumn{1}{c|}{ Terminology used in EBD paper }} & Clinical* & Non-clinical & \multirow{2}{*}{ t - value } & \multirow{2}{*}{ p - value } \\
\cline { 2 - 3 } & Mean \pm SD & Mean \pm SD & & \\
\hline Relative risk & $2.70 \pm 0.78$ & $2.55 \pm 0.77$ & 1.29 & 0.11 \\
\hline Absolute risk & $2.60 \pm 0.74$ & $2.85 \pm 0.81$ & 2.21 & $0.02^{*}$ \\
\hline Systematic review & $2.85 \pm 0.81$ & $2.68 \pm 0.79$ & 1.41 & 0.15 \\
\hline Clinical effectiveness & $2.72 \pm 0.79$ & $2.63 \pm 0.76$ & 0.77 & 0.44 \\
\hline Meta-analysis & $2.58 \pm 0.78$ & $2.50 \pm 0.67$ & 0.73 & 0.48 \\
\hline Number needed to treat & $2.57 \pm 0.75$ & $2.58 \pm 0.69$ & 0.09 & 0.93 \\
\hline Odds ratio & $2.51 \pm 0.68$ & $2.43 \pm 0.67$ & 0.79 & 0.43 \\
\hline Sensitivity and specificity & $2.79 \pm 0.75$ & $2.65 \pm 0.68$ & 1.28 & 0.21 \\
\hline Confidence interval & $2.42 \pm 0.74$ & $2.18 \pm 0.53$ & 2.31 & $0.02^{*}$ \\
\hline Publication bias & $2.59 \pm 0.74$ & $2.52 \pm 0.68$ & 0.64 & 0.52 \\
\hline Sample bias & $2.73 \pm 0.77$ & $2.58 \pm 0.70$ & 1.33 & 0.18 \\
\hline
\end{tabular}

*Independent samples $\mathrm{t}$-test $(\mathrm{p}<0.05)$ 
Table 3 - Terminologies that needed additional information by the study subjects

\begin{tabular}{|l|c|c|}
\hline $\begin{array}{c}\text { Terminology used in EBD } \\
\text { paper }\end{array}$ & N & \% \\
\hline Confidence interval & 128 & 54.7 \\
\hline Odds ratio & 104 & 44.4 \\
\hline Meta-analysis & 102 & 43.6 \\
\hline Number needed to treat & 96 & 41 \\
\hline Publication bias & 95 & 40.6 \\
\hline Clinical effectiveness & 91 & 38.9 \\
\hline Absolute risk & 82 & 35 \\
\hline Sampling bias & 73 & 31.2 \\
\hline Sensitivity and specificity & 72 & 30.8 \\
\hline Systematic review & 72 & 30.8 \\
\hline Relative risk & 69 & 29.5 \\
\hline
\end{tabular}

Attitude towards practice of evidence based dentistry among the study subjects was shown in figure 2. More than $60 \%$ of the study subjects agreed to the fact that evidence based dentistry bring about quick knowledge update, helps in clinical decision making, improves the quality of patient care, reduce health care costs and evidence based dentistry should be an integral part of the dental school curriculum. Negative attitude towards practice of evidence based dentistry was evident among $10 \%$ of the study population.

Figure 2 - Attitude towards practice of Evidence Based Dentistry

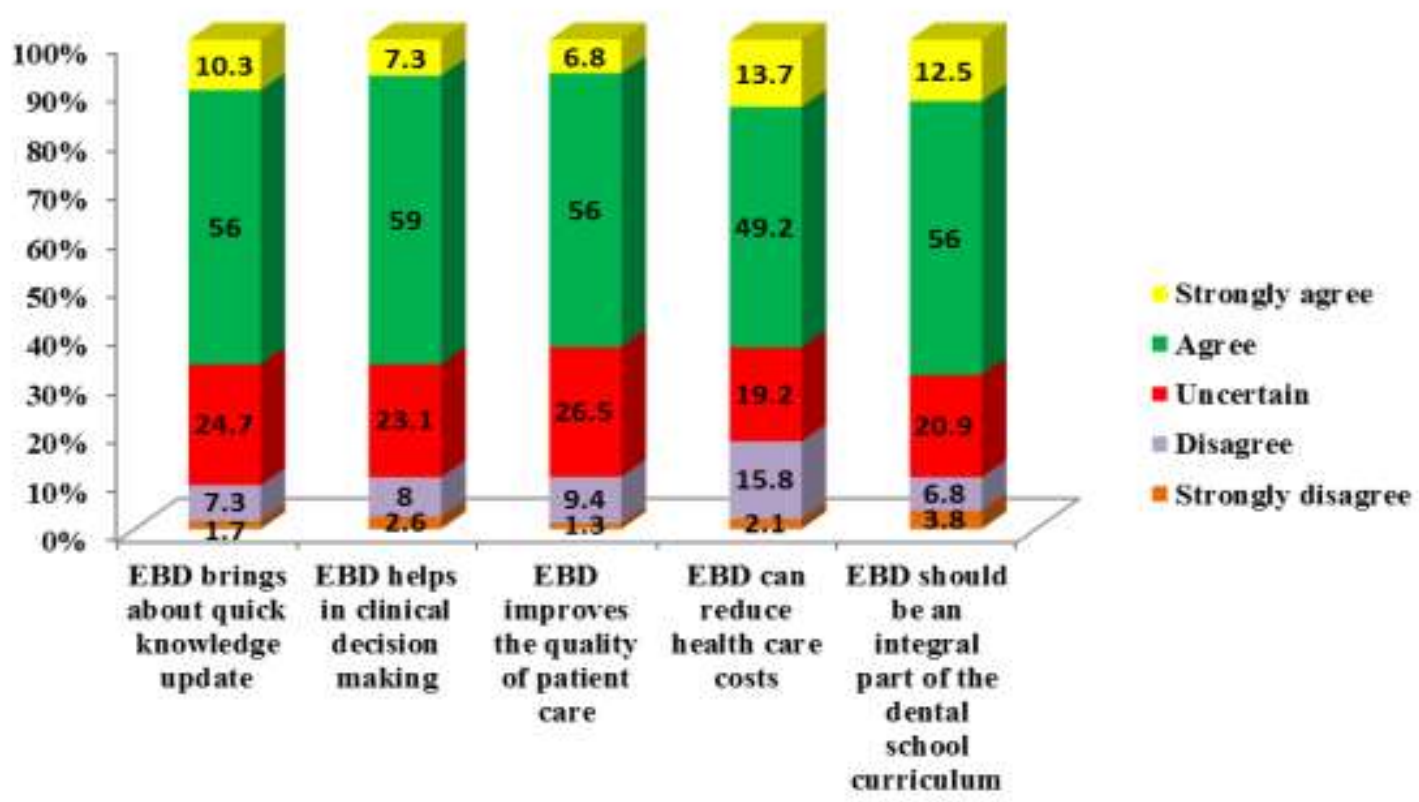

Even though a positive attitude was seen among the post graduates in practice of evidence based dentistry, there exist certain barriers in practice of evidence based dentistry depicted in figure 3. Lack of skills to appraise scientific journals, financial constraints and difficulties in application into routine clinical practice were considered as the major barriers towards practice of evidence based dentistry. 
Figure 3 - Potential Barriers towards practice of Evidence based Dentistry

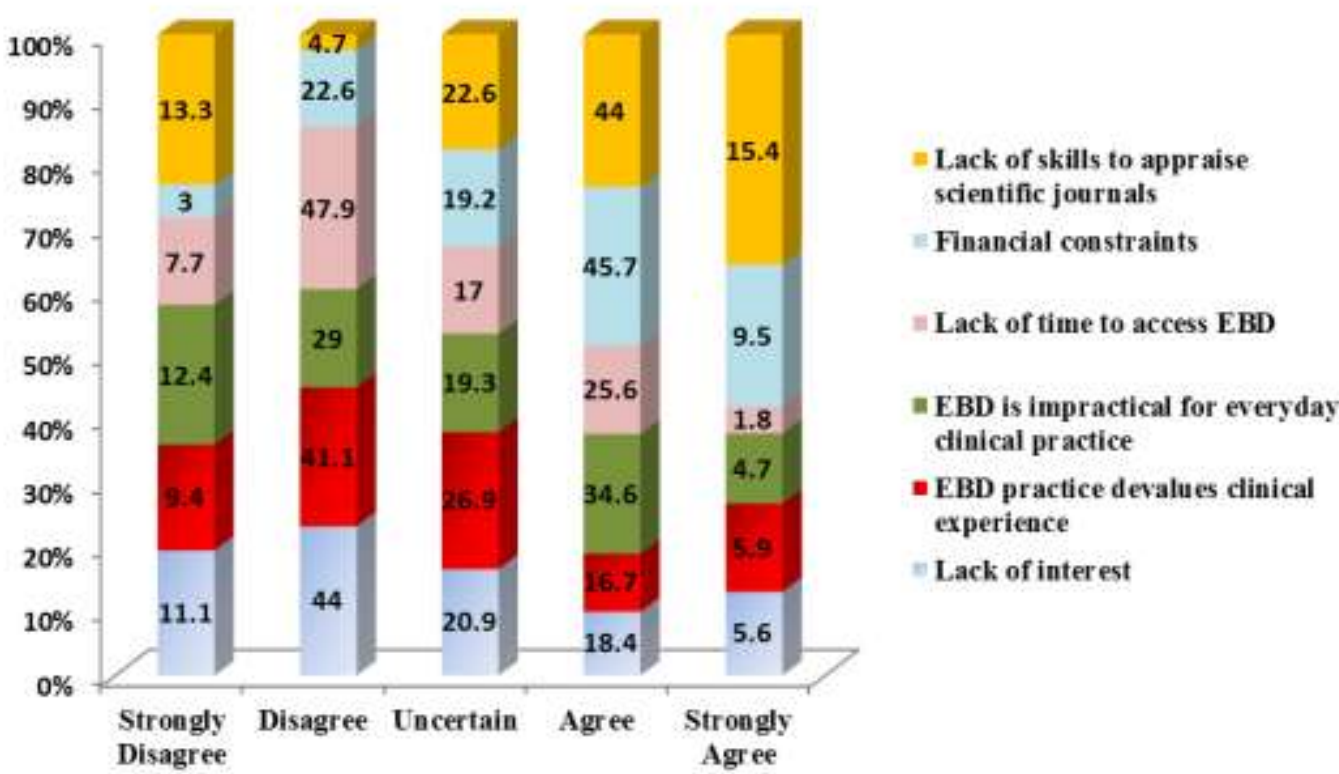

IV. Discussion

Evidence based dentistry is said to be the current best approach to provide interventions as it improves dentist's skills and knowledge as well as quality of treatment provided to the patients. Research works in various branches of dentistry is on the rise in India, mainly by the postgraduate dental students and by the faculties of the various dental colleges, there is a need to update their clinical knowledge to improve their research outcomes. Evidence based dentistry is the solution to provide such updates. Currently there is a lack of studies to assess the knowledge, attitude and perceived barriers towards practice of Evidence based Dentistry among postgraduates, hence this cross-sectional questionnaire survey was done to assess the knowledge, attitude and perceived barriers towards practice of evidence based dentistry among postgraduates.

In the present study more than $70 \%$ of the respondents were familiar with the sources of Evidence based dentistry; this was in accordance with the study by Zamros Y.M. et al (2008) ${ }^{[15]}$. This finding is important and it reflects an essential quality of a modern day clinicians in which the changing socio-demographic patterns of the population and knowledgeable consumers have resulted in high demands for best practice and clinical decision making. In the current study 27.8\% respondents were unaware of Evidence based dental practice from BDJ publishing group, this was higher than the study by Zamros Y.M. et al (2008) ${ }^{[15]}$ where only $2.2 \%$ were unaware of Evidence based dental practice, the difference was due to the difficulty in accessing evidence based dental practice due to financial constraints. In contrast, the present study showed that only $30.8 \%$ respondents were unaware of Cochrane database for systematic reviews, lower than the study by Zamros Y.M et al (2008) ${ }^{[15]}$ where $58.5 \%$ respondents were unaware of Cochrane database for systematic reviews, Cochrane collaboration provides free online access to their published systematic reviews hence this database is widely accessed by the postgraduates. In the present study $10.3 \%$ respondents were unaware of Pubmed / Medline journals; this was in accordance with the study by Risahmawati RM Risahmawati et al (2011) ${ }^{[13]}$.

The key tool in the evidence based approach is the systematic literature review for their explicit, welldocumented, scientific methodology in order to reduce errors or biases and to provide a more, objective, comprehensive view of the research literature ${ }^{[16]}$. Regarding, the terminologies used in evidence based dentistry paper; majority of the respondents had some understanding of the terminologies used in the paper. The learning of skills needed to critically appraise scientific articles is central to the practice of evidence based dental practice ${ }^{[17]}$ present study indicated that the respondents understood many of the terms, on the other hand the respondents still wanted to know more about the terms like confidence interval, Odds ratio, Metaanalysis, comparatively higher than the study by Risahmawati RM et al (2011) ${ }^{[13]}$.

In the present study more than $60 \%$ respondents agreed to the fact that evidence based dental practice brings quick knowledge update, helps in clinical decision making, improves patient care, reduce health care costs and evidence based dentistry should be a part of dental school curriculum, the findings are in accordance with the study by Sowmya Anaberu Rajasekaran et al (2011) ${ }^{[10]}$ and Zamros Y.F et al (2008) ${ }^{[15]}$. This positive attitude should be looked upon as an opportunity to identify weakness and promote understanding of the concept 
of evidence based dentistry by conducting seminars on evidence based dentistry or by recommending the introduction of evidence based practice into the dental education curriculum.

In terms of perceived barriers in use of evidence based dentistry, financial constraints followed by difficulties in application into routine clinical practice are the most common barriers, this finding was in accordance with the study by Zamros Y.F et al (2008) ${ }^{[15]}$ and Iqbal and Glenny (2002) ${ }^{[2]}$. The present study showed that the postgraduates lack adequate skills to appraise scientific journals, this was due to limited knowledge of the terminologies used in evidence based dentistry and the findings are in accordance to the study results where the post graduates seek more information on the terminologies used in evidence based dentistry paper, dental curriculum should be modified to overcome this barrier.

The limitations of the present study was that in this cross-sectional survey the responses were participants self-report and self-judgement, the frequency of use of these electronic database for solving the clinical problems were not recorded, also the other aids used by the postgraduates to resolve their clinical problems encountered were not recorded. Further studies are required to assess the use of other sources of information in resolving clinical problems among postgraduates, similar assessment is required among the faculties of various dental colleges and a special training program on evidence based dentistry has to be developed for the postgraduates to impart evidence based dentistry into their clinical practice.

\section{Conclusion}

Evidence based dentistry is relatively a new paradigm in dentistry and thus may not be a well-known concept to every dental graduate. The present study showed that the postgraduates although familiar with the sources of evidence based dentistry, their knowledge of the terms used in evidence based dentistry is limited and they seek more information about the terminologies, this was reflected as a positive attitude of the respondents towards imparting evidence based dentistry in clinical practice and the respondents agreed to include evidence based practice in dental curriculum. Potential barriers exist in practice of evidence based dentistry.

There should be an adequate program developed in the form research workshops and seminars on evidence based dentistry to overcome the barriers perceived by the postgraduates in practice of evidence based dentistry, thereby integrating the concept of evidence based dentistry into routine clinical practice, which improves the quality of dental care provided to the patient .

\section{References}

[1] Choudry NK, Fletcher RH and Soumerai SB. Systematic review: The relationship between clinical experience and quality of healthcare. Annals of Internal Medicine 2005:142(260):73-94.

[2] A. Iqbal and AM. Glenny. General dental practitioners' knowledge of and attitude towards evidence-based practice. British Dental Journal 2002:192:587-591.

[3] Bader J, Ismail A. Survey of systematic reviews in Dentistry. Journal of American Dental Association 2004:135:464-473.

[4] Winning T, Needleman I, Rohlin M. Evidence based care and the curriculum. European Journal of Dental Education 2008:12(sup 1):48-63.

[5] Hyanes B, Haynes GA. What does it take to put an ugly face through the heart of a beautiful hypothesis? (Editorial). Annals of Internal Medicine 2009: 150:JC3-2-3.

[6] Rosenberg W, Donald A. Evidence-based medicine: an approach to clinical problem-solving. British Medical Journal 1995:310:1122-1126.

[7] Kay EJ, Blinkhorn AS. A qualitative investigation of factors governing dentists' treatment philosophies. British Dental Journal 1996:180:171-176.

[8] Lockwood S. 'Evidence of me' in evidence based medicine? British Medical Journal 2004:329:1033-1035.

[9] Shelja Vashisth, Mohit Bansal, Nidhi Gupta and Nanak Chand Rao. Evidence Based Dentistry an evolving concept. Journal of the Indian Association of Public Health Dentistry. 2011:18:226-228.

[10] Sowmya Anaberu Rajshekar, Sunder Kote K and Lakshminarayan Nagesh. Knowledge and Attitude towards Evidence based Dentistry among Postgraduate students of two Dental Colleges in Davangere City. Journal of the Indian Association Public Heal th Dentistry 2011:18(sup-II):761-765

[11] Durgesh N Balloor. Evidence based education in Dentistry: Can it be implemented in India? International Journal of Oral and Maxillofacial Pathology 2011; 2(2): 44-45.

[12] I.M. Haron, M.Y. Sabti and R. Omar. Awareness, knowledge and practice of evidence - based dentistry amongst dentists in Kuwait. European Journal of Dental Education 2012; 16(1):e47-e52.

[13] Risahmawati RM Risahmawati, Sei SE Emura, Tomoko TN Nishi and Shunzo SK Koizumi. Japanese Resident Physicians' attitude, knowledge and perceived barriers on the practice of Evidence based Medicine: a survey. BMC Research notes 2011; $4: 374$.

[14] Hendricson WD, Rugh JD, Hatch JP, Stark DL, Deahl T and Wallmann ER. Validation of an instrument to assess evidence-based practice knowledge, attitudes, access ad confidence in the dental environment. Journal of Dental Education 2011; 75(2): 131-144.

[15] Zamros Y.M, Ha LJ, San PP and Ramli AS. Evidence-based practice among a group of Malaysian dental practitioners. Journal of Dental Education 2008; 72(11): 1333-1342.

[16] Mulrow CD. The medical review article: state of the science. Annals of Internal Medicine 1987; 106:485-488.

[17] Sackett DL, Haynes RB. On the need for evidence-based medicine. Evidence based Medicine 1995; 1:5-6. 